\title{
Influence of falling height on the excitability of the soleus $H$-reflex during drop-jumps
}

\author{
C. Leukel,' W. Taube,' M. Gruber,' M. Hodapp' and A. Gollhofer' \\ 1 Department of Sport Science, University of Freiburg, Freiburg, Germany \\ 2 Department of Clinical Neurology and Neurophysiology, University of Freiburg, Freiburg, Germany
}

Correspondence: C. Leukel, Department of Sport Science, University of Freiburg, Schwarzwaldstr. 175, 79117 Freiburg. Germany. E-mail: christian.leukel@sport.unifreiburg.de

\begin{abstract}
Aim: The stretch-shortening cycle (SSC) is characterized by stretching of the target muscle (eccentric phase) prior to a subsequent shortening in the concentric phase. Stretch reflexes in the eccentric phase were argued to influence the performance of short lasting SSCs. In drop-jumps, the short latency component of the stretch reflex (SLR) was shown to increase with falling height. However, in jumps from excessive heights, the SLR was diminished. So far, it is unclear whether the modulation of the SLR relies on spinal mechanisms or on an altered fusimotor drive. The present study aimed to assess the spinal excitability of the soleus Ia afferent pathway at SLR during jumps from low height $(\mathrm{LH}-31 \mathrm{~cm})$ and excessive height $(\mathrm{EH}-76 \mathrm{~cm})$.

Methods: In 20 healthy subjects (age $25 \pm 3$ years), H-reflexes were timed to occur at the peak of the SLR during drop-jumps from LH and EH.

Results: H-reflexes were significantly smaller at $\mathrm{EH}$ than at $\mathrm{LH}(P<0.05)$. Neither soleus and tibialis anterior background EMG nor the size of the maximum $\mathrm{M}$-wave changed with falling height.

Conclusion: Differences in the H-reflex between $\mathrm{EH}$ and $\mathrm{LH}$ indicate that spinal mechanisms are involved in the modulation of the SLR. A decreased excitability of the H-reflex pathway at EH compared with LH is argued to serve as a 'prevention strategy' to protect the tendomuscular system from potential injuries caused by the high load. It is argued that pre-synaptic inhibition of Ia afferents is most likely responsible for the change in H-reflex excitability between the two jump conditions.
\end{abstract}

Keywords drop-jump, H-reflex, stretch-shortening cycle.
The stretch-shortening cycle (SSC) is an efficient muscular activation type characterized by stretching of the target muscle (eccentric phase) prior to a subsequent shortening in the concentric phase. When compared with concentric actions without preceding stretch, muscular force and power of the SSC is enhanced (Cavagna et al. 1965, Gregor et al. 1988). In short lasting SSCs during running, hopping or in drop-jumps, the short latency response (SLR) of the spinal stretch reflex was argued to be essential for the improved performance (Dietz et al. 1979, Gollhofer et al. 1992, Voigt et al. 1998, Komi 2000, Trimble et al. 2000, Funase et al. 2001). The size of the SLR was shown to depend on the velocity of the applied muscle stretch (Gollhofer \& Rapp 1993). In dropjumps, the SLR showed a higher amplitude with increased drop height from 20 to $40 \mathrm{~cm}$ and to $60 \mathrm{~cm}$ respectively (Komi \& Gollhofer 1997). However, in jumps from excessive heights $(80 \mathrm{~cm})$, the SLR was diminished. Despite larger impact loads and higher stretch velocities, the neuromuscular activation was decreased. This observation was argued to represent a protective inhibition to reduce eccentric stress on the tendomuscular system (Schmidtbleicher \& Gollhofer 
1982, Kyrolainen \& Komi 1995, Komi \& Gollhofer 1997). Decreased Ia afferent input arising from muscle spindles and/or an increased inhibitory drive from various sources such as Golgi tendon organs or voluntary protection mechanisms were suggested (Komi \& Gollhofer 1997). Changes in the excitability of the Ia afferent pathway could occur on the spinal level or could be induced by an altered fusimotor drive. Regarding the amount of fusimotor drive, differences were shown during functional tasks like walking, balancing or landing in animals (Hulliger et al. 1989). Due to methodological problems, it is difficult to assess the amount of fusimotor drive during functional tasks in humans yet. During landing, fusimotor activity was highest when the animal was in midair and declined upon landing (Hulliger et al. 1989). To our knowledge, there is no study which investigated fusimotor drive during jumping.

Spinal modulation of Ia afferent input in humans was shown in studies investigating functional tasks, like gait (Faist et al. 1996, Simonsen \& DyhrePoulsen 1999). If such a modulation is effective to control Ia afferent input during automatic movements, it can be hypothesized that similar mechanisms may also affect the SLR in voluntary movements like drop-jumps. On the spinal level, changes in reflex excitability can be assessed by the H-reflex transmitted via a similar pathway as the short latency stretch reflex but bypassing the muscle spindles. The aim of this study was to compare H-reflex excitability during the SLR component in the landing phase of drop-jumps from excessive and normal heights. It was hypothesized that H-reflex excitability is reduced at excessive heights compared with normal heights.

\section{Material and methods}

\section{Subjects}

A total of 20 trained students (age $25 \pm 3$ years) without any history of orthopaedic or neurological disorder participated. The term trained subjects in this context refers to students who exercised on a regular basis in sports activities which contain jump exercises (volleyball, basketball). Trained subjects were selected because they (1) showed less variation with respect to the performance of drop-jumps than untrained subjects, (2) were less fatigable than untrained subjects and (3) exhibited shorter contact times in drop-jumps than untrained subjects which is essential in investigating short lasting SSC activities. The study was approved by the local ethics committee and all subjects gave informed consent.

\section{Main protocol}

Experimental setup. A total of 10 subjects (age $25 \pm 4$ years) participated in this part of the study. Drop-jumps had to be performed from two different heights, $31 \mathrm{~cm}$ (low height, LH) and $76 \mathrm{~cm}$ (excessive height, EH). These specific heights were chosen based on theoretical assumptions from a preliminary study because it was important that the background EMG of the soleus muscle (SOL) at SLR was matched between $\mathrm{LH}$ and $\mathrm{EH}$ to allow for comparison of H-reflex amplitudes. It was previously shown that the SLR increased in size with increased falling height in dropjumps and decreased again after more than $60 \mathrm{~cm}$ drop height (Komi \& Gollhofer 1997).

After a warm-up (hopping) of $3 \mathrm{~min}$, subjects had first to get customized to the jumping procedure. Before jumping, both arms had to be held akimbo (hands at the ilium), the left leg remained stable to secure upright stance whereas the right leg was lifted and projected in front of the body. The jump off was performed with the left leg. The purpose of this procedure was to keep the right leg (from which was recorded) relaxed until jump off. Subjects were asked to touch down on both feet and to jump off the ground as quickly as possible. Additionally, subjects were instructed to keep the jumping procedure similar throughout all jumps with regard to starting position on the platform, head position and push off phase after landing. The position of each subject on the platform before jumping was visually controlled by an experimenter. Trials were repeated if the starting position was altered.

After subjects got customized to the jumping procedure, EMG of SOL and gastrocnemius medialis (GM) from $10 \mathrm{SSCs}$ at $\mathrm{LH}$ was rectified and averaged to determine individual stimulation instants (Fig. 1, see below 'Stimulation parameters and $\mathrm{H}$-reflexes'). Thereafter, subjects had to execute drop-jumps with tibial nerve stimulation from $\mathrm{LH}$ and EH. A total of 120 jumps were performed. Subjects were instructed to rest for $2 \mathrm{~min}$ after every 10 jumps to avoid fatigue.

EMG recordings. EMG recordings were obtained from the SOL, tibialis anterior (TA) and GM muscles of the right leg. After preparation, cups of the $\mathrm{Ag}-\mathrm{AgCl}$ surface electrodes (Marquette Hellige ${ }^{\circledR}$, Freiburg, Germany, diameter $9 \mathrm{~mm}$ ) were filled with electrode jelly and attached to the skin above the muscle belly in a longitudinal fashion (inter-electrode distance $2 \mathrm{~cm}$ ). The reference electrode was placed on the tibial plateau. Impedance had to be below $5 \mathrm{k} \Omega$. All electrodes were checked for movement artefacts by actively shaking the right leg. EMG signals were sampled at $4 \mathrm{kHz}$, amplified $(\times 1000)$ and bandpass-filtered $(30-1000 \mathrm{~Hz})$. The 

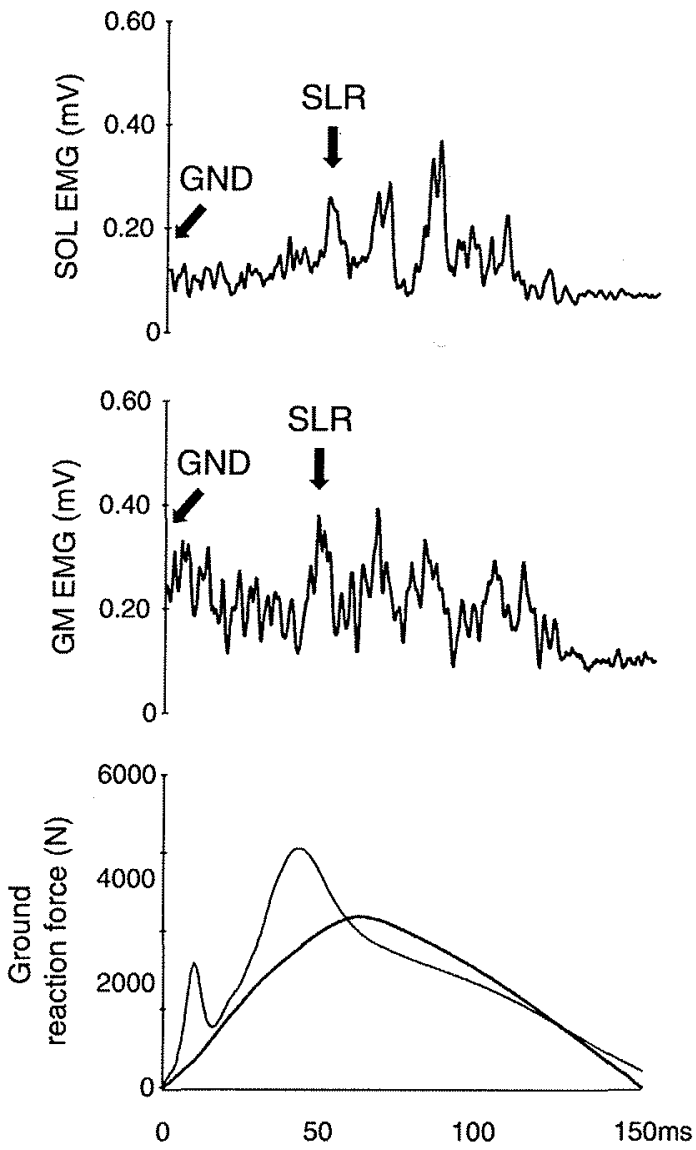

Figure I SLR was determined from rectified and averaged EMGs of SOL during landing at LH. Stimulation was triggered, so that the peak of the $\mathrm{H}$-reflex coincided either with GND or SLR (black arrows). The thick line of ground reaction force represents $\mathrm{LH}$. The thin line represents $\mathrm{EH}$ and was superimposed to show differences in ground reaction force between $\mathrm{LH}$ and $\mathrm{EH}$.

EMGs were stored on a hard drive for later analysis with custom built software (LABVIEW ${ }^{\circledast}$ based; National Instruments, Austin, TX, USA).

Stimulation parameters and H-reflexes. For each subject, two stimulation instants were determined during the drop-jump cycle. Stimulation was timed, so that the peak of the $\mathrm{H}$-reflex coincided with one of the stimulation instants. Either the peak of the H-reflex coincided with ground contact (GND, first stimulation instant) or with the peak of the short latency reflex response in SOL (SLR, second stimulation instant, see also Fig. 1). Spinal excitability at GND was assessed as a reference to SLR. A light barrier mounted $25 \mathrm{~cm}$ above the ground was used to trigger the H-reflex at GND. A trigger signal was initiated when subjects passed the barrier. H-reflex stimulation was applied after a defined delay with respect to the trigger signal. This delay was calculated according to the individual latency of the H-reflex and the individual time between passing the barrier and ground contact. For the latter, individual time between passing the barrier and ground contact was calculated at $\mathrm{LH}$ and $\mathrm{EH}$ during jumps without stimulation. Onset of the vertical force triggered the H-reflex at the SLR via the force platform. Again, the delay between the trigger signal and $\mathrm{H}$-reflex stimulation was calculated according to the individual latency of the H-reflex and the individual latency of SLR. For instance, a subject with an $\mathrm{H}$-reflex latency of $40 \mathrm{~ms}$ and a latency of the SLR of $48 \mathrm{~ms}$ was stimulated with a delay of $8 \mathrm{~ms}$ after the trigger was activated by the force platform.

H-reflexes were elicited with an electrical stimulator (AS100; Alea Solutions ${ }^{\circledR}$, Zurich, Switzerland) in the right SOL muscle by stimulating the posterior tibial nerve. Stimuli consisted of square-wave pulses of $1 \mathrm{~ms}$ duration. The anode, a $10 \times 5 \mathrm{~cm}$ dispersal pad, was fixed on the anterior aspect of the knee just underneath the patella. During upright stance, the cathode $(2 \mathrm{~cm}$ in diameter) was placed in the popliteal fossa and moved stepwise until the optimum position for eliciting an $\mathrm{H}$-reflex was found. Care was taken that the stimulation did not activate TA. H/M recruitment curves were recorded during quiet stance to preset the stimulus intensity during drop-jumps. During drop-jumps, a previously developed stimulation method (Voigt et al. 1998, Simonsen \& Dyhre-Poulsen 1999, Ferris et al. 2001) was used to (1) ascertain constant stimulation intensity at each stimulation instant, (2) to adjust the stimulus strength to fluctuations of the maximal $\mathrm{M}$-wave during the drop-jump and (3) to avoid fatigue of the subjects by reducing the total experimental time (Simonsen \& Dyhre-Poulsen 1999). This method was developed to test the spinal excitability of the H-reflex pathway during dynamic movements like walking, running and hopping, and is therefore suitable to test the spinal excitability of Ia afferents during the highly dynamic condition drop-jump in this study. For each stimulation instant, we used two electrical stimuli with an interstimulus-interval of $60 \mathrm{~ms}$. The first stimulus was timed, so that the peak of the H-reflex coincided with the defined stimulation instant (GND or SLR). The second stimulus was applied $60 \mathrm{~ms}$ after the first one. For both of these stimuli, an $\mathrm{M}$-wave was visible in the SOL EMG (see Fig. 2). The second $\mathrm{M}$-wave was elicited with supramaximal stimulation intensity [two times the intensity used to elicit the maximum $M$-wave (Mmax) during upright stance]. The following procedure was performed for each jump cycle: a custom built computer program $\left(\right.$ LABviEW $^{\circledR}$ ) triggered the stimuli and recorded the amplitude of both $\mathrm{M}$-waves. According to the methods described by Voigt et al. (1998) the amplitude of the first $M$-wave (first electrical stimulus) was 


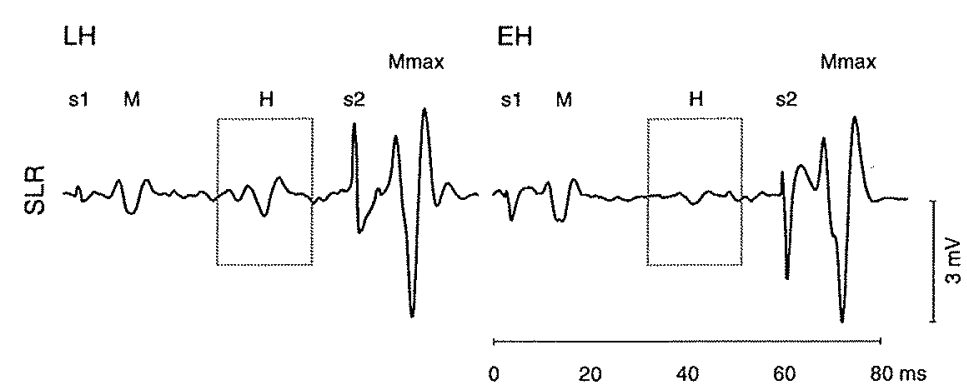

Figure $2 \mathrm{H}$-reflex modulation (mean) in a single subject at SLR in the LH and EH condition. Each trace shows, from left to right: $s 1$, the first stimulus artefact; $M$, the first $M$-wave; $H$, the $H$-reflex; $s 2$, the second stimulus artefact and Mmax, the second $\mathrm{M}$-wave. The second stimulus was elicited $60 \mathrm{~ms}$ after the first stimulus. The latency of the H-reflex (peak) in this subject was $42 \mathrm{~ms}$.

adjusted to $25 \pm 10 \%$ compared with the second Mmax (range between $15 \%$ and $35 \%$ of Mmax). The program calculated the ratio between the first and the second $\mathrm{M}$-wave, stored the sweep if the requirements were fulfilled or deleted the sweep if the first M-wave did not match the set criteria. If the sweep had to be deleted, the stimulation intensity for the first stimulus was adjusted. If the sweep could be stored, the stimulus intensity was retained.

\section{Additional protocol}

Ten subjects (age $24 \pm 2$ years) participated in the additional protocol. The experimental setup was the same as in the main protocol. In the additional protocol, $\mathrm{H} / \mathrm{M}$ recruitment curves were recorded at $\mathrm{LH}$ and $\mathrm{EH}$. However, to reduce the number of jumps, H-reflexes were only recorded at the SLR stimulation instant. In this experimental setup, ankle angles (dorsalflexion and plantarflexion) and knee angles (knee flexion and extension) were recorded during drop-jumps. After the jumping procedure, ankle and knee angles at SLR were calculated from jumps at LH and EH (see 'Data analysis and statistics'). Subjects were instructed to reproduce respective knee and ankle angles from jumps of the two conditions in a standing position and $\mathrm{H} / \mathrm{M}$ recruitment curves were recorded. It was ensured that SOL and TA background EMG was the same between the two standing conditions.

Data analysis and statistics. H-reflexes and $M$-waves were expressed as peak to peak amplitudes. In the main protocol, $\mathrm{H}$-reflexes in all conditions were accepted if the first $M$-wave amplitude was in the range of $15-35 \%$ of the following Mmax amplitude. Given this range and the fact that small changes in the $\mathrm{M}$-wave could equate to large changes in the $\mathrm{H}$-reflex amplitude, it may be possible that changes in the $\mathrm{H}$-reflex between $\mathrm{LH}$ and $\mathrm{EH}$ could be related to changes in stimulation intensity and not to differences in falling height. To account for this, M-wave/Mmax ratios were calculated for each trial in all conditions. Thereby, the $\mathrm{M}$-wave was expressed as a percentage of the corresponding Mmax. The ratios were compared between heights at GND and SLR. Trials were accepted if pairs in the ratio could be found of $\mathrm{LH}$ and $\mathrm{EH}$ trials which were not separated by more than $\pm 3 \%$. For instance, at SLR a subject showed ratios of $30.2 \%, 25.1 \%, 22.4 \%, 29.1 \%$ at $\mathrm{LH}$ and of $33.1 \%$, $30.1 \%, 16.2 \%, 25.2 \%$ at $\mathrm{EH}$. The following pairs and therefore trials were accepted: $30.2 \%$ and $33.1 \%$, $25.1 \%$ and $25.2 \%, 29.1 \%$ and $30.1 \%$. Hmax/Mmax ratios (H/M ratios) were calculated from peak to peak amplitude $\mathrm{H}$-reflexes and $\mathrm{M}$-waves. In the main protocol, H-reflexes at SLR were compared with $\mathrm{H}$-reflexes recorded in upright stance $(\mathrm{H} / \mathrm{M}$ recruitment curves). In upright stance, only $\mathrm{H}$-reflexes with the same $\mathrm{M}$-waves as for the SLR stimulation instant were selected for analysis. To analyse the corresponding background $\mathrm{EMG}$, a 20 -ms time window was set around GND and SLR in which root mean square (RMS) values were calculated. RMS background EMG was calculated from jumps without electrical stimulation. Ankle and knee angles at SLR were calculated as RMS values in an $8-\mathrm{ms}$ time window around the SLR stimulation instant. $180^{\circ}$ knee angle refers to full knee extension. An angle of $90^{\circ}$ between the fifth metatarsal and the fibula is defined as $90^{\circ}$ ankle angle. All values are expressed as mean \pm standard deviation $(S D)$ if not indicated differently.

A repeated measures ANOVA procedure with the factors of stimulation instant (GND and SLR) and falling height ( $\mathrm{LH}$ and $\mathrm{EH}$ ) was conducted to reveal differences in H-reflex excitability and background EMG in the main protocol. In case of significant F-values $(P<0.05)$ differences between values at each stimulation instant were compared by a paired twosided test (Student's $t$-test). Paired Student's $t$-tests were also used to indicate differences of all other variables in the main and additional protocol between LH and EH. 
Differences were regarded significant at $P<0.05$ for all tests.

\section{Results}

\section{Main protocol}

The $\mathrm{H}$-reflex excitability was significantly different $(P<0.05)$. H-reflex amplitudes recorded at SLR were lower at $\mathrm{EH}$ than at $\mathrm{LH}(P<0.05$, Fig. 3$)$ whereas there were no differences at GND (not significant, ns). SOL and TA background EMG were not significantly different (ns) whereas GM revealed differences $(P<0.05)$. In terms of the distinct stimulation instants, $G M$ was not significantly different at SLR (ns) whereas there was a difference at GND $(P<0.05)$.

$\mathrm{H} / \mathrm{M}$ ratios obtained during upright stance were $0.42 \pm 0.12$. The latency of SLR (referring to ground contact) was $51 \pm 4 \mathrm{~ms}$. Due to the defined criterion of acceptable trials, a mean \pm SD of $5 \pm 1.2$ trials were analysed for GND and $5 \pm 1.5$ for SLR.
There was no difference in Mmax between $\mathrm{EH}$ and LH (ns) for stimulation at GND and also at SLR (Fig. 3). H-reflexes in stance were $1.43 \pm 0.94 \mathrm{mV}$ and thus significantly decreased if compared with SLR in the $\mathrm{LH}$ condition $(2.01 \pm 1.21 \mathrm{mV}, P<0.05)$. There were no differences if $\mathrm{H}$-reflexes during stance were compared with the SLR in the $\mathrm{EH}$ condition $(1.54 \pm 1.03 \mathrm{mV}, \mathrm{ns})$.

\section{Additional protocol}

At SLR, subjects showed decreased $\mathrm{H} / \mathrm{M}$ ratios at $\mathrm{EH}$ $(0.46 \pm 0.16)$ compared with LH $(0.61 \pm 0.24$, $P<0.01$; Fig. 4). Ankle angles at SLR were significantly smaller at EH $\left(74.3 \pm 10^{\circ}\right)$ than at $\mathrm{LH}\left(79.7 \pm 11.3^{\circ}\right.$, $P<0.01)$. Knee angles at SLR did not differ between the two conditions (EH: $131.4 \pm 13.6^{\circ}$; $\mathrm{LH}: 134.8 \pm$ $\left.17^{\circ}, \mathrm{ns}\right) . \mathrm{H} / \mathrm{M}$ recruitment curves were additionally recorded in a standing condition in which ankle and knee angles were reproduced according to the joint angles in jumps from $\mathrm{EH}$ and LH at SLR. Interestingly,
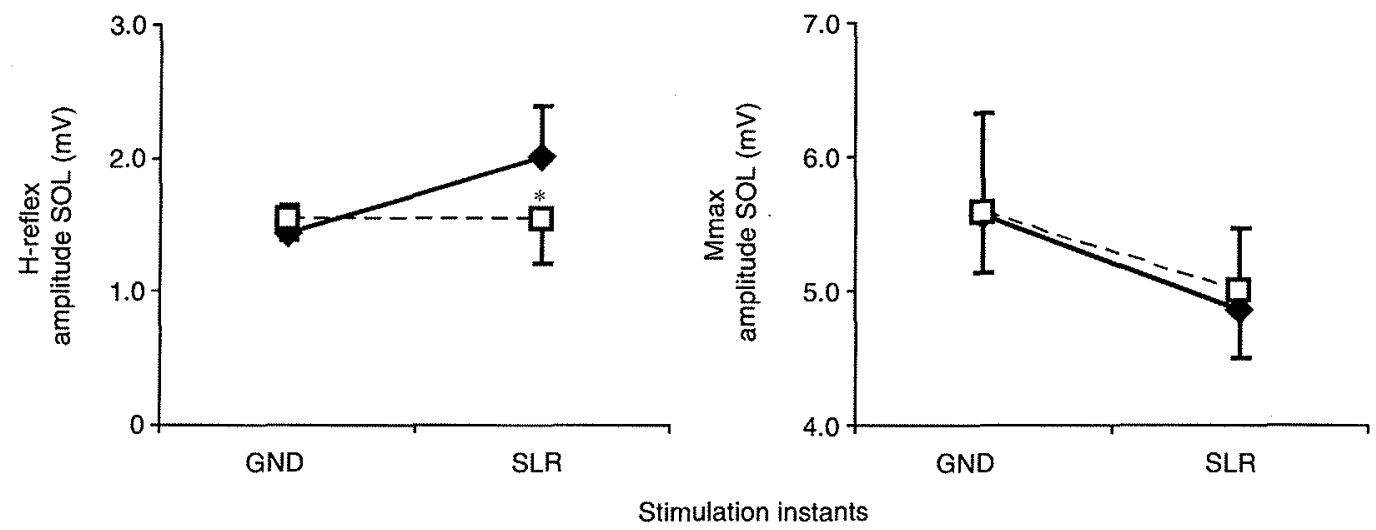

Figure 3 Comparison of SOL H-reflex amplitudes (mean) between LH $(\bullet)$ and EH $(\square)$ on the left side and Mmax (right side) at GND and SLR. Black bars indicate standard error of mean (SEM) $(* P<0.05)$.

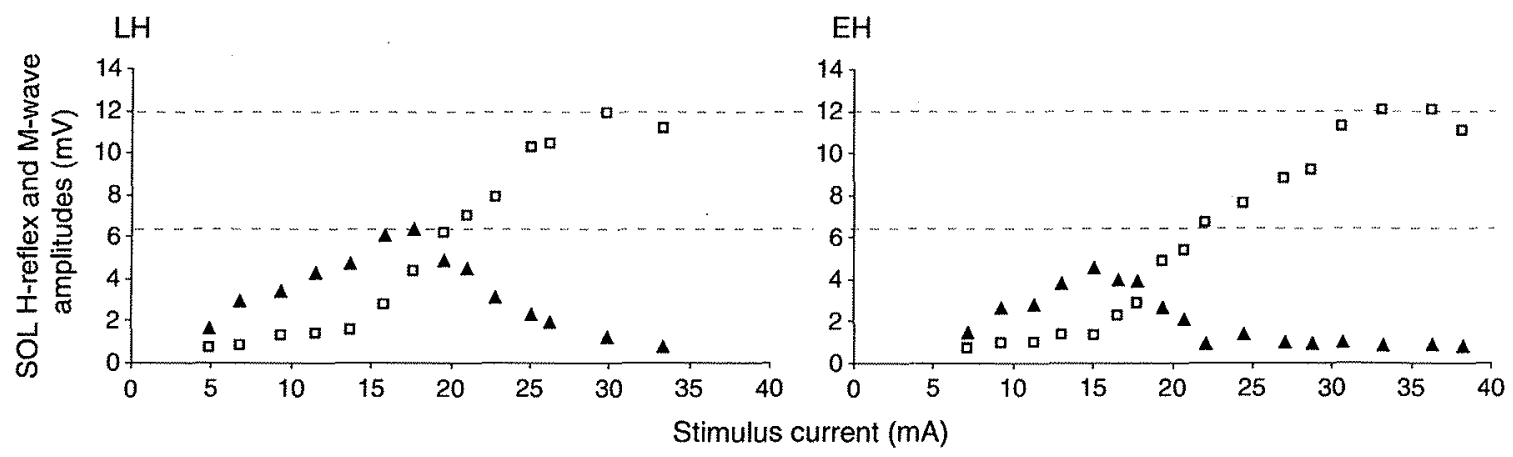

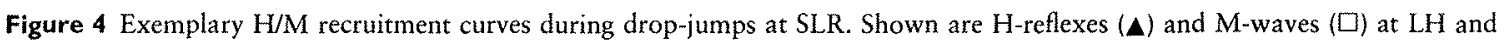
EH. Dashed lines indicate Hmax at LH and Mmax at LH respectively. 
there were no differences in the H/M ratios between the two conditions (EH: $0.42 \pm 0.15 ; \mathrm{LH}: 0.4 \pm 0.15$, ns).

\section{Discussion}

The aim of the present study was to investigate modulations in the transmission of Ia afferents with regard to falling height in drop-jumps. Based on previous studies (Schmidtbleicher \& Gollhofer 1982, Kyrolainen \& Komi 1995, Komi \& Gollhofer 1997), these modulations were expected at SLR. SLR showed a latency of $51 \pm 4 \mathrm{~ms}$ in this study and, therefore, fit well to the latency of the mainly monosynaptic component of the stretch reflex described in the literature (Thilmann et al. 1991, Taube et al. 2006).

H-reflex excitability was the same at GND but was significantly lower at SLR at EH than at $\mathrm{LH}$ in the main protocol. SOL increased its activity from GND to SLR. One would assume that SOL H-reflex excitability increases with a higher muscular activation. Interestingly, this was only the case at $\mathrm{LH}$ whereas at $\mathrm{EH}$ the $H$-reflexes remained on a similar level from GND to SLR.

\section{Methodological considerations}

Stimulation intensities to elicit $\mathrm{H}$-reflexes were high in the main protocol. Thereby, $\mathrm{H}$-reflexes could have been stimulated on the descending limb of the H/M recruitment curve in these subjects (Crone et al. 1990). Here, $\mathrm{H}$-reflexes are mainly susceptible to inhibition. In this study, inhibition of the SOL H-reflex at SLR at EH compared with LH was assumed. Therefore, the methodology was adequate to test the hypothesis although $\mathrm{H}$-reflexes were stimulated on the descending limb of the H/M recruitment curve. Furthermore, H/M recruitment curves were recorded at SLR at LH and EH in the additional protocol. Here, H/M ratios were significantly lower at $\mathrm{EH}$ than at $\mathrm{LH}$.
Fatigue could have changed the H-reflex. However, fatigue is supposed to be negligible in the present experiment. A protocol similar to that of our study with similar subjects did not show changes in maximum voluntary contraction torque (MVC) of the ankle plantarflexors, SOL EMG activity in the eccentric and concentric phase, take-off velocity and contact time before and after executing drop-jumps (Avela et al. 2006). The instruction in the study of Avela et al. (2006) was to jump maximal. The instruction in the present experiment was just to keep the jumping procedure similar throughout all jumps without focussing on maximum jump performance.

Altered ankle joint position could change the amplitude of the $\mathrm{H}$-reflex by changing the recording EMG electrodes with respect to the underlying muscle fibres (Gerilovsky et al. 1989). Indeed, different ankle joint angles at SLR between $\mathrm{LH}$ and EH were shown in the additional protocol. However, the different ankle angle at SLR between $\mathrm{LH}$ and EH seems not to be responsible in modulating SOL H-reflex excitability during drop-jump. H/M recruitment curves were recorded during upright stance with the same ankle and knee angles as in the $\mathrm{LH}$ and $\mathrm{EH}$ jumping condition. There were no differences in the H/M ratios in the stance conditions. In contrast, there were differences in the H/M ratios at SLR during drop-jumps between $\mathrm{EH}$ and LH. Therefore, spinal adaptations seem to be involved in the control of the H-reflex and, consequently, in the transmission of la afferents in dropjumps from different falling heights. However, changes in fusimotor drive cannot be excluded.

\section{Modulation of transmission of la afferents at SLR}

Falling heights were chosen to match background EMG of SOL (and TA) at SLR (Fig. 5). Therefore, postsynaptic modulation via $\mathrm{Ib}$ inhibition or reciprocal inhibition in these muscles seem unlikely. It was shown
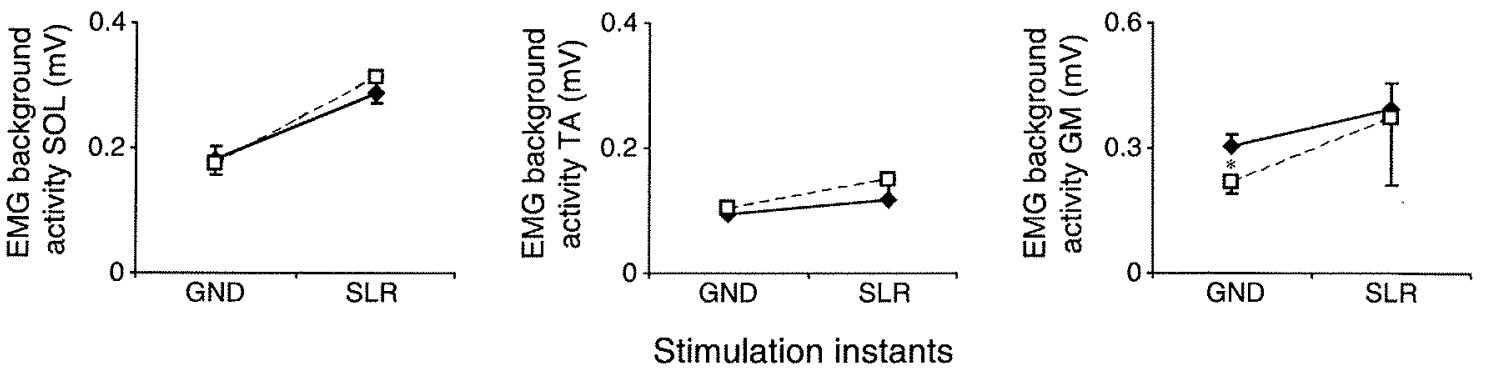

Figure 5 Comparison of the background EMG in SOL (left side), TA (middle) and GM (right side) between LH ( $\bullet$ ) and EH ( $\square$ ) at GND and SLR. The high variability of the GM background EMG at SLR was caused by one subject who showed extensively high muscular activation compared with the other subjects. Note that EMGs were calculated from jumps without electrical stimulation. Black bars indicate standard error of mean (SEM) ("P $<0.05)$. 
that $\mathrm{Ib}$ inhibition of GM onto SOL can reduce SOL H-reflexes (Pierrot-Deseilligny et al. 1981). Therefore, it could be speculated that Ib inhibition from GM influenced the SOL $\mathrm{H}$-reflex at SLR because of different activation levels of GM at GND (Fig. 5). However, as GM activity at GND was lower at $\mathrm{EH}$ than at $\mathrm{LH}$, increased SOL $\mathrm{H}$-reflexes would be expected at $\mathrm{EH}$ rather than at LH. As post-synaptic mechanisms fail to explain the H-reflex modulation in our study it is suggested that the H-reflex size was changed by pre-synaptic mechanisms. Rhythmic modulation of pre-synaptic inhibition of la afferents was shown to be effective to modulate $\mathrm{H}$-reflexes during walking (Faist et al. 1996, Simonsen \& Dyhre-Poulsen 1999). Accordingly, pre-synaptic inhibition may also be a good candidate in modulating Ia afferent input between LH and $\mathrm{EH}$ drop-jumps in the present study. Different descending and ascending pathways are capable to transmit pre-synaptic inhibition. Considering the ascending pathways, activation of peripheral afferents (e.g. cutaneous afferents) are capable to alter the level of pre-synaptic inhibition of Ia afferents (Iles 1996). However, cutaneous as well as other peripheral afferents with conduction velocities slower than Ia afferents are not assumed to change the H-reflex at SLR in our study due to temporal constraints: The mechanical stimulus at ground contact is thought to activate muscle spindle afferents rapidly. As Ia afferents are the fastest transmitting afferent fibres available (Duysens et al. 2002), it is unlikely that other peripheral mechanisms triggered at ground contact - are capable to transmit their signals faster to the spinal cord. Activation of peripheral afferents preceding the impact is also unlikely to modulate Ia afferents on the spinal level because there is no stimulus which could activate peripheral receptors. Furthermore, the EMG activity immediately preceding impact was argued to be 'preprogrammed' in origin and not to arise from a reflex (Jones \& Watt 1971). Therefore, it is reasonable to assume that descending pathways control the transmission of Ia pre-synaptic inhibition. Supraspinal structures are known to interact with spinal mechanisms during movement (Prochazka 1989, Hultborn 2001). H-reflexes were shown to increase before voluntary contraction of a muscle (Burke et al. 1992) and also if a movement was imagined without visible skeletomotor activity (Gandevia et al. 1997). The importance of supraspinal centres in changing the muscular activity during jumping was emphasized by McDonagh \& Duncan (2002). In line with McDonagh \& Duncan (2002), it is assumed that supraspinal centres determine the amount of spinal excitability of la afferents at SLR in the present study. It may be speculated that from a functional point of view, reduction of the Ia afferent input at $\mathrm{EH}$ could serve as a prevention strategy due to the high load. Diminished reflexes could have reduced the stiffness of the tendomuscular system and therefore reduced the strain (Hoffer \&. Andreassen 1981, DyhrePoulsen et al. 1991).

\section{Comparison of GM background EMG between $L H$ and EH}

At GND, GM showed significantly lower activation at EH than at LH (Fig. 5). In drop-jumps, high activation of GM at ground contact can prevent yielding of the contractile elements of a muscle (Komi 2003). This activation is necessary for efficient storage of elastic energy in the muscle-tendon complex and thus potentiates performance in the subsequent shortening phase of the drop-jump (Komi 2003). However, if the drop-jump was performed from an excessive height, muscular activity at GND was probably suppressed to prevent the tendomuscular system from high loads.

\section{Conclusions}

When falling height in drop-jumps was increased, $\mathrm{H}$-reflexes in SOL at SLR were significantly decreased. Spinal excitability of Ia afferents is thought to be lower at $\mathrm{EH}$ than at $\mathrm{LH}$ as a 'prevention strategy' to protect the tendomuscular system from potential injuries due to the high load. As the most likely mechanism in modulating la afferent input, pre-synaptic inhibition of la afferents is argued to adjust the spinal gating according to the falling height. It is suggested that changes in pre-synaptic inhibition at SLR are controlled by supraspinal mechanisms.

\section{Conflict of interest}

There are no conflicts of interest.

The authors wish to thank Dr Michael Faist for his helpful comments on the manuscript. This study was supported by a grant of BISP (Bundesinstitut fuer Sportwissenschaft).

\section{References}

Avela, J., Finni, J. \& Komi, P.V. 2006. Excitability of the soleus reflex arc during intensive stretch-shortening cycle exercise in two power-trained athlete groups. Eur J Appl Physiol 97, 486-493.

Burke, D., Gracies, J.M., Meunier, S. \& Pierrot-Deseilligny, E. 1992. Changes in presynaptic inhibition of afferents to propriospinal-like neurones in man during voluntary contractions. J Physiol 449, 673-687.

Cavagna, G.A., Saibene, F.P. \& Margaria, R. 1965. Effect of negative work on the amount of positive work performed by an isolated muscle. $J$ Appl Physiol 20, 157-158. 
Crone, C., Hultborn, H., Mazieres, L., Morin, C., Nielsen, J. \& Pierrot-Deseilligny, E. 1990. Sensitivity of monosynaptic test reflexes to facilitation and inhibition as a function of the test reflex size: a study in man and the cat. Exp Brain Res 81, $35-45$.

Dietz, V., Schmidtbleicher, D. \& Noth, J. 1979. Neuronal mechanisms of human locomotion. I Neurophysiol 42, 1212-1222.

Duysens, J., Van de Crommert, H.W., Smits-Engelsman, B.C. \& Van der Helm, F.C. 2002. A walking robot called human: lessons to be learned from neural control of locomotion. J Biomech 35, 447-453.

Dyhre-Poulsen, P., Simonsen, E.B. \& Voigt, M. 1991. Dynamic control of muscle stiffness and $\mathrm{H}$ reflex modulation during hopping and jumping in man. $J$ Physiol 437, 287-304.

Faist, M., Dietz, V. \& Pierrot-Deseilligny, E. 1996. Modulation, probably presynaptic in origin, of monosynaptic la excitation during human gait. Exp Brain Res 109, 441-449.

Ferris, D.P., Aagaard, P., Simonsen, E.B., Farley, C.T. \& Dyhre-Poulsen, P. 2001. Soleus H-reflex gain in humans walking and running under simulated reduced gravity. J Physiol 530, 167-180.

Funase, K., Higashi, T., Sakakibara, A., Imanaka, K., Nishihira, Y. \& Miles, T.S. 2001. Patterns of muscle activation in human hopping. Eur J Appl Physiol 84, 503-509.

Gandevia, S.C., Wilson, L.R., Inglis, J.T. \& Burke, D. 1997. Mental rehearsal of motor tasks recruits alpha-motoneurones but fails to recruit human fusimotor neurones selectively. J Physiol 505, 259-266.

Gerilovsky, L., Tsvetinov, P. \& Trenkova, G. 1989. Peripheral effects on the amplitude of monopolar and bipolar H-reflex potentials from the soleus muscle. Exp Brain Res 76, 173-181.

Gollhofer, A. \& Rapp, W. 1993. Recovery of stretch reflex responses following mechanical stimulation. Eur J Appl Physiol Occup Physiol 66, 415-420.

Gollhofer, A., Strojnik, V., Rapp, W. \& Schweizer, L. 1992. Behaviour of triceps surae muscle-tendon complex in different jump conditions. Eur J Appl Physiol Occup Physiol 64, 283-291.

Gregor, R.J., Roy, R.R., Whiting, W.C., Lovely, R.G., Hodgson, J.A. \& Edgerton, V.R. 1988. Mechanical output of the cat soleus during treadmill locomotion: in vivo vs in situ characteristics. J Biomech 21, 721-732.

Hoffer, J.A. \& Andreassen, S. 1981. Regulation of soleus muscle stiffness in premammillary cats: intrinsic and reflex components. I Neurophysiol 45, 267-285.

Hulliger, M., Durmuller, N., Prochazka, A. \& Trend, P. 1989. Flexible fusimotor control of muscle spindle feedback during a variety of natural movements. Prog Brain Res 80, 87-101.
Hultborn, H. 2001. State-dependent modulation of sensory feedback. J Physiol 533, 5-13.

Iles, J.F. 1996. Evidence for cutaneous and corticospinal modulation of presynaptic inhibition of Ia afferents from the human lower limb. J Pbysiol 491, 197-207.

Jones, G.M. \& Watt, D.G. 1971. Observations on the control of stepping and hopping movements in man. J Physiol 219 , 709-727.

Komi, P.V. 2000. Stretch-shortening cycle: a powerful mode! to study normal and fatigued muscle. J Biomech 33, 11971206.

Komi, P.V. 2003. Stretch-shortening cycle. In: P.V. Komi (ed.) Strength and Power in Sport, pp. 184-202. Blackwell Science, Oxford.

Komi, P.V. \& Gollhofer, A. 1997. Stretch reflex can have an important role in force enhancement during SSC-exercise. $J$ Applied Biomech 13, 451-460.

Kyrolainen, H. \& Komi, P.V. 1995. Differences in mechanical efficiency between power- and endurance-trained athletes while jumping. Eur J Appl Physiol Occup Physiol 70, 36-44.

McDonagh, M.J. \& Duncan, A. 2002. Interaction of pre-programmed control and natural stretch reflexes in human landing movements. J Physiol 544, 985-994.

Pierrot-Deseilligny, E., Morin, C., Bergego, C. \& Tankov, N. 1981. Pattern of group I fibre projections from ankle flexor and extensor muscles in man. Exp Brain Res 42, $337-350$.

Prochazka, A. 1989. Sensorimotor gain control: a basic strategy of motor systems? Prog Neurobiol 33, 281-307.

Schmidtbleicher, D. \& Gollhofer, A. 1982. Neuromuskuläre Untersuchungen zur Bestimmung individueller Belastungsgrößen für ein Tiefsprungtraining. Leistungssport 12, 298-307.

Simonsen, E.B. \& Dyhre-Poulsen, P. 1999. Amplitude of the human soleus $\mathrm{H}$ reflex during walking and running. $J$ Pbysiol .515, 929-939.

Taube, W., Schubert, M., Gruber, M., Beck, S., Faist, M. \& Gollhofer, A. 2006. Direct corticospinal pathways contribute to neuromuscular control of perturbed stance. $J$ Appl Pbysiol 101, 420-429.

Thilmann, A.F., Schwarz, M., Topper, R., Fellows, S.J. \& Noth, J. 1991. Different mechanisms underlie the longlatency stretch reflex response of active human muscle at different joints. J Physiol 444, 631-643.

Trimble, M.H., Kukulka, C.G. \& Thomas, R.S. 2000. Reflex facilitation during the stretch-shortening cycle. J Electromyogr Kinesiol 10, 179-187.

Voigt, M., Dyhre-Poulsen, P. \& Simonsen, E.B. 1998. Modulation of short latency stretch reflexes during human hopping. Acta Physiol Scand 163, 181-194. 